ARTICLE

\title{
Acetylation of MAT Il $\alpha$ represses tumour cell growth and is decreased in human hepatocellular cancer
}

Hong-Bin Yang ${ }^{1, \star}$, Ying-Ying $X u^{1, \star}$, Xiang-Ning Zhao ${ }^{1,2}$, Shao-Wu Zou ${ }^{2}$, Ye Zhang ${ }^{1}$, Min Zhang ${ }^{1}$, Jin-Tao Li ${ }^{3}$, Feng Ren', Li-Ying Wang ${ }^{1} \&$ Qun-Ying Lei ${ }^{1,3,4}$

Metabolic alteration is a hallmark of cancer. Dysregulation of methionine metabolism is implicated in human liver cancer. Methionine adenosyltransferase $\| \alpha(M A T \| \alpha)$ is a key enzyme in the methionine cycle, catalysing the production of S-adenosylmethionine (SAM), a key methyl donor in cellular processes, and is associated with uncontrolled cell proliferation in cancer. Here we show that P300 acetylates MAT $\| \alpha$ at lysine residue 81 and destabilizes MAT $\| \alpha$ by promoting its ubiquitylation and subsequent proteasomal degradation. Conversely, histone deacetylase- 3 deacetylates and stabilizes MAT Il $\alpha$ by preventing its proteasomal degradation. Folate deprivation upregulates $\mathrm{K} 81$ acetylation and destabilizes MAT $\| \alpha$ to moderate cell proliferation, whereas a single mutation at K81 reverses the proliferative disadvantage of cancer cells upon folate deprivation. Moreover, MAT Il $\alpha$ K81 acetylation is decreased in human hepatocellular cancer. Collectively, our study reveals a novel mechanism of MAT $\| \alpha$ regulation by acetylation and ubiquitylation, and a direct functional link of this regulation to cancer development.

\footnotetext{
${ }^{1}$ Key Laboratory of Metabolism and Molecular Medicine, Ministry of Education, and Department of Biochemistry and Molecular Biology, School of Basic Medical Sciences, Fudan University, Shanghai 200032, China. ${ }^{2}$ Department of Hepatopancreatobiliary Surgery, Shanghai 10th People's Hospital, Tong Ji University, Shanghai 200072, China. ${ }^{3}$ Cancer Metabolism Lab, Institutes of Biomedical Sciences, Fudan University, Shanghai 200032 , China. ${ }^{4}$ Collaborative Innovation Center of Systems Biomedicine, Shanghai Jiaotong University, Shanghai 200025, China. ${ }^{\star}$ These authors contributed equally to this work. Correspondence and requests for materials should be addressed to Y.Y.X. (email: yingyingxu@fudan.edu.cn) or to S.W.Z (email: shaowuzou@126.com) or to Q.Y.L. (email: qlei@fudan.edu.cn).
} 
F olate is essential for rapidly proliferating cells and plays a pivotal role in one-carbon metabolism. Its metabolic derivative tetrahydrofolate (FH4), known as the carrier of one-carbon units, is involved in de novo synthesis of thymidylate and purine, amino-acid inter-conversion and so on ${ }^{1}$. Coupling with methionine cycle, which produces the major methyl donor $S$-adenosylmethionine (SAM), folate metabolism also presents methyl-groups to global methylation of macromolecules.

Methionine adenosyltransferase (MAT) is the key enzyme in methionine cycle, catalysing biosynthesis of SAM. In mammals, two distinct genes, MAT1A and MAT2A, encode two homologous catalytic subunits $-\alpha 1$ and $\alpha 2$, respectively ${ }^{2}$. MAT1A mainly expresses in healthy hepatocytes. Its encoding product, the $\alpha 1$ subunits, assemble into two MAT isozymes, MAT III (dimer) and MAT I (tetramer) ${ }^{2}$. In contrast, MAT $2 A$ and its coding product, MAT II $\alpha$ (dimer formed from $\alpha 2$ subunits), is widely expressed in all exhepatic tissues at relatively low level under normal conditions $^{3}$, whereas overexpressed in various human epithelial tumours, including gastric, colon and liver cancer ${ }^{4-7}$.

Studies indicated that enhanced MAT2A expression boosts cancer cell proliferation and potentially promotes tumour development and progression ${ }^{2,8,9}$. In addition, MAT2A, but not $M A T 1 A$, is found expressed in fetal liver, whereas healthy adult liver expresses mainly $M A T 1 A^{3}$. MAT expression is progressively switched from MAT2A to MAT1A during liver development, whereas is reversed to $M A T 2 A$ again during liver malignant transformation. In addition, knockdown MAT2A dramatically suppresses tumour cell proliferation and induces cell cycle arrest and apoptosis ${ }^{7,10,11}$. Therefore, the transcriptional switch from $M A T 1 A$ to MAT2A is believed to play a role in facilitating cancer cell survival and proliferation ${ }^{11,12}$.

MAT2A expression is regulated at both transcriptional and post-transcriptional levels. Sp1 (transcription factor: specificity protein 1), c-Myb (transcription activator: avian myeloblastosis viral oncogene), NF- $\kappa \mathrm{B}$ (nuclear factor of kappa, enhancer in B cells) and AP-1 (adaptor-related protein complex 1) were identified as the trans-activating factors involved in MAT2A transcriptional upregulation ${ }^{13,14}$. Tumour-necrosis factor- $\alpha$ upregulates MAT2A via NF- $\kappa \mathrm{B}$ and AP-1 (ref. 14). Moreover, histone acetylation, promoter methylation and mRNA stabilization were also reported to regulate MAT2A expression $^{15}$. Although regulation of $M A T 2 A$ expression was heavily studied at transcriptional and post-transcriptional levels, its post-translational regulation remains largely unknown.

Covalent lysine acetylation has been identified as an evolutionarily conserved modification in metabolic enzymes, and plays critical roles in regulation of multiple enzymes ${ }^{16-18}$. In this study, we demonstrate that MAT II $\alpha$ protein is acetylated at lysine residue 81 in response to folate deprivation. Acetylation promotes MAT II $\alpha$ ubiquitylation and its subsequent proteasomal degradation, inhibiting tumour cell proliferation. Our observations reveal a novel mechanism of MAT II $\alpha$ upregulation in human cancers.

\section{Results}

MAT II $\alpha$ is acetylated at lysine 81. Previous mass spectrometry (MS) analyses indicated that MAT II $\alpha$ was potentially an acetylated protein (Supplementary Fig. 1a). To confirm the acetylation modification, pan acetyl-lysine antibody was used to detect the acetylation level of ectopically expressed MAT II $\alpha$. Result showed that MAT II $\alpha$ was indeed acetylated in HEK293T and Chang's cells (Fig. 1a). Furthermore, trichostatin A (TSA), an inhibitor of histone deacetylase HDAC family I, II and IV, increased the acetylation level of flag-MAT II $\alpha$ approximately two- to threefold (Fig. 1a). Given that lysine (K) 81 is the only putative acetylation site identified in MAT II $\alpha$ by MS (Fig. $1 \mathrm{~b}$ and Supplementary Fig. 1a), and is evolutionarily conserved from D. melanogaster to mammals, we mutated K81 to arginine (R) and glutamine (Q) and found that both mutations resulted in a significant reduction in acetylation (Fig. 1c). Notably, TSA treatment dramatically increased the acetylation level of wild-type MAT II $\alpha$, but not K81R or K81Q mutant, indicating K81 might be the primary acetylation site of MAT II $\alpha$ (Fig. 1c). To further confirm acetylation of K81, we generated a K81 site-specific antibody specifically targeting to acetylated K81 residue in MAT II $\alpha$ (designated as 'K81Ac antibody' henceforth). Dot blotting assay was performed to characterize the specificity of this antibody and found that K81Ac antibody preferentially detected K81 acetylated, but not unmodified peptide (Fig. 1d). In addition, strong and specific signal of K81 acetylation was observed in ectopically expressed wild-type MAT II $\alpha$ but not K81R mutant (Supplementary Fig. 1b). Further results showed that both MAT2A knockdown (Fig. 1e) and peptide blocking by K81 acetylated peptide (Fig. 1f) significantly reduced K81Ac signal, indicating a high specificity of this antibody to MAT II $\alpha$ K81 acetylation. More importantly, with the help of this site-specific antibody, we detected enhanced K81 acetylation signal of endogenous MAT II $\alpha$ in different cell lines upon TSA treatment (Fig. 1g). These results indicate that $\mathrm{K} 81$ is the major acetylation site of MAT II $\alpha$ under the tested condition.

Folate decreases K81 acetylation and stabilizes MAT II $\alpha$. Folate's metabolic derivative tetrahydrofolate plays an essential role in transferring one-carbon units. Restoration of methionine from homocysteine in methionine cycle greatly depends on methyl-groups (one type of one-carbon units) presented from folate metabolism. Therefore, methionine cycle is tightly related to folate availability and one-carbon unit metabolism. As serine hydroxymethyl transferation and glycine cleavage are two major sources of one-carbon units, methionine cycle is also related to serine and glycine metabolisms. To determine whether K81 acetylation of MAT II $\alpha$ is dynamically regulated by serine, glycine or folate in vivo, we cultured HEK293T cells in medium containing these metabolites with different concentrations and found that K81 acetylation level of MAT II $\alpha$ increased as concentrations of folate decreased (Fig. 2a, upper panel). In contrast to this, serine and glycine showed no effect on MAT II $\alpha$ K81 acetylation (data not shown). More interestingly, steady-state level of MAT II $\alpha$ protein decreased as concentrations of folate decreased (Fig. 2a, lower panel). Similar results were obtained in human hepatocellular carcinoma cell line-Huh7 (Supplementary Fig. 2a). These results indicate a potential inverse correlation between MAT II $\alpha$ acetylation and protein levels in response to folate concentration.

K81 acetylation promotes MAT II $\alpha$ degradation. To determine whether downregulation of MAT II $\alpha$ protein by folate occurs at transcriptional level, quantitative reverse transcription-PCR was performed. Results showed that folate treatment had no effect on MAT II $\alpha$ mRNA level (Fig. $2 b$ ), indicating that folate regulates MAT II $\alpha$ protein mainly at the post-transcriptional level. To determine whether acetylation induced MAT II $\alpha$ degradation is mediated by proteasomal pathway, we treated HEK293T cells with proteasome inhibitor MG132 and found both TSA and folate deprivation induced decrease in MAT II $\alpha$ protein can be readily restored by MG132 (Fig. 2c and Supplementary Fig. 2b), suggesting K81 acetylation in response to TSA or folate deprivation might target MAT II $\alpha$ protein to proteasomal degradation. The fact that MAT II $\alpha$ was accumulated in HEK293T, H1299 and U937 cells after treated with MG132 further supported this 
a

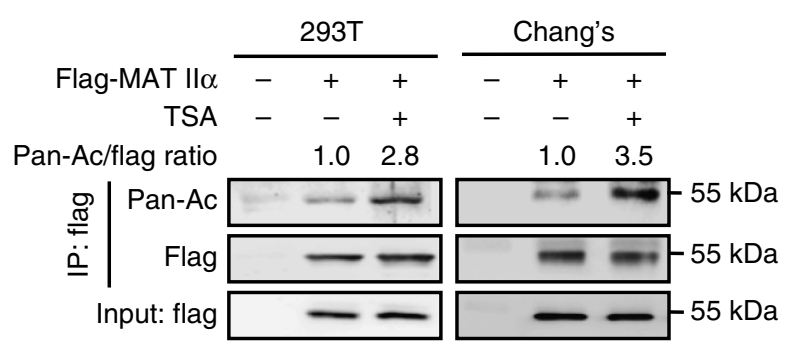

b

Hs: AAVDYQKVVR
Pt:AAVDYQKVVR
Cl:AAVDYQKVVR
Bt:AAVDYQKVVR
$M m:$ AAVDYQKVVR
Rn:AAVDYQKVVR
Dm:AAVDYQKVVR
C

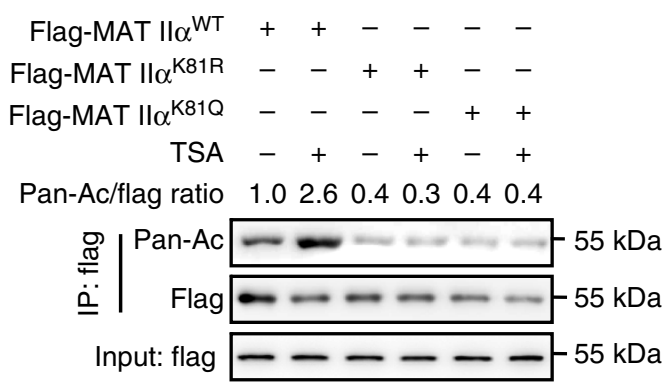

d

Amount peptide (ng) $500 \quad 200 \quad 100 \quad 50$
K81Ac-peptide
Unmodified peptide

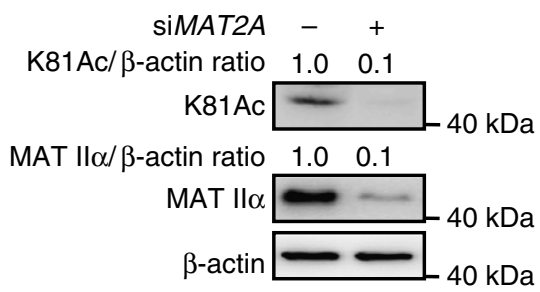

$\mathbf{f}$

\begin{tabular}{|c|c|c|c|}
\hline K81Ac antibody & + & + & + \\
\hline MAT II $\alpha$ antibody & - & - & - \\
\hline K81Ac peptide & - & + & - \\
\hline K81 peptide & - & - & + \\
\hline Relative intensity & $\begin{array}{ll}1.0 & 0.8\end{array}$ & 0.1 & 1.2 \\
\hline
\end{tabular}

g

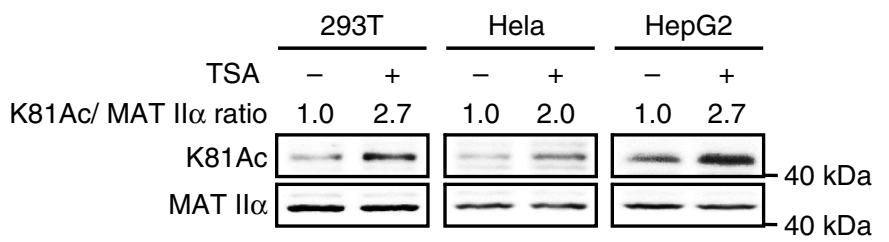

Figure 1 | MAT Il $\alpha$ is acetylated at lysine 81. (a) Exogenous MAT Il $\alpha$ is acetylated. Flag-MAT Il $\alpha$ was transfected into HEK293T and Chang's cells followed by TSA treatment $(10 \mu \mathrm{M})$ for $18 \mathrm{~h}$. Flag-MAT $\| \alpha$ acetylation was detected by anti-acetyllysine (Pan-Ac) antibody. (b) Alignment of MAT Il $\alpha$ lysine 81 and adjacent protein sequence from different organisms. Hs: Homo Sapient (human); Pt: Pan troglodytes (chimpanzee); Cl: Canis lupus familiaris (dog); Bt: Bos taurus (bull); Mm: Mus musculus (mouse); Rn: Rattus norvegicus (Norway rat); Dm: Drosophila melanogaster (fruit fly). (c) TSA increases acetylation level of wild-type MAT $\| \alpha$ but not mutants. Ectopically expressed wild-type MAT $\| \alpha$, K81R and K81Q were transfected into HEK293T cells followed by TSA treatment, MAT Il $\alpha$ acetylation was analysed by western blotting. (d) Specificity of $K 81$ site-specific acetylation antibody was determined by dot blotting assay. (e) MAT2A knockdown confirmed the K81Ac antibody detection. HEK293T cells were transfected with MAT2A siRNA or scramble control and cell lysates were subjected to western blotting. Signal detected by K81Ac antibody decreased as MAT Il $\alpha$ was knocked down. (f) K81Ac antibody is specific to MAT Il $\alpha$ K81 acetylation. HEK293T cells were lysed and directly subjected to western blotting. The four repetitively loaded lanes were clipped into four membranes and were exposed to antibodies as indicated, separately. For peptide block, acetylated K81 peptide (the antigen we used for K81Ac antibody generation) or non-acetylated K81 peptide (mimicking MAT Il $\alpha$ protein) were added to the diluted K81Ac antibodies (1:200) in a final concentration of $300 \mu \mathrm{g} \mathrm{ml}^{-1}$, cultivated at $37^{\circ} \mathrm{C}$ for $1 \mathrm{~h}$ before applying to membranes. Four clipped membranes were aligned and exposed together when developing film to make sure their relative signal intensities are comparable. (g) TSA promotes MAT Il $\alpha$ acetylation at K81 in different cell lines. HEK293T, Hela and HepG2 cells were treated as indicated. K81 acetylation was detected by K81Ac antibody and normalized against MAT II $\alpha$ protein.

speculation (Supplementary Fig. 2c). In addition, TSA treatment combined with protein synthesis inhibitor cycloheximide (CHX) dramatically decreased the half-life of endogenous MAT II $\alpha$ (Supplementary Fig. 2d). These results indicate that TSA- and folate deprivation-induced MAT II $\alpha$ protein degradation is proteasomal dependent. To further test the possibility that TSA or folate deprivation promotes MAT II $\alpha$ degradation via K81 acetylation, half-lives of wild-type, K81R and K81Q MAT II $\alpha$ were determined by CHX chase experiment. Wild-type MAT II $\alpha$, but not the mutants, was found to display a shortened half-life under folate-deprived condition (Fig. 2d), indicating proteasomal degradation of MAT II $\alpha$ might be related to K81 acetylation. In addition, we employed ubiquitylation ladder assay and observed a significant increase in wild-type MAT II $\alpha$ ubiquitylation in the presence of TSA or upon folate deprivation, whereas ubiquitylation of K81R mutation was mostly blocked and no longer respond to either TSA or folate deprivation (Fig. 2e,f). Consistently, we found MG132 could block TSA- or folate deprivation-induced MAT II $\alpha$ destabilization (Fig. $2 \mathrm{~g}$ and Supplementary Fig. 2e). Collectively, these results demonstrate that K81 acetylation of MAT II $\alpha$ promotes its degradation via ubiquitylation-mediated proteasomal degradation.

UBR4 targets MAT II $\alpha$ for degradation. Three types of enzymes are required in the process of ubiquitylation: ubiquitin-activating enzymes (E1s), ubiquitin-conjugating enzymes (E2s) and ubiquitin-ligases (E3s), among which, only E3s define the target specificity of ubiquitylation reaction. To further investigate the mechanism of TSA- or folate deprivation-induced MAT II $\alpha$ 
proteasomal degradation, we established an HEK293-derived cell line stably expressing both streptavidin-binding protein and flagtagged MAT II $\alpha$, cultured upon folate deprivation and purified MAT II $\alpha$ protein using tandem affinity purification to identify its E3 ligase via MS. Among the consistently identified interacting proteins, we identified UBR4 (ubiquitin protein ligase E3 component n-recognin 4, belongs to the zinc finger E3 ligase family) as a putative E3 ligase for MAT II $\alpha$. To verify whether UBR4 is an
E3 ligase for MAT II $\alpha$, we overexpressed UBR4 (D) (expressing a truncated form of UBR4 protein, with substrate-binding and catalytic domains only) in HEK293T cells and found a significant drop in MAT II $\alpha$ protein, which was totally reversed by adding MG132 to the culture medium (Fig. 3a). Furthermore, knocking down UBR4 expression by three independent short interfering RNAs (siRNAs) could instantly block folate deprivation-induced MAT II $\alpha$ degradation (Fig. 3b). Consistently, CHX chase a

Folate $\left(\mathrm{mg} \mathrm{L}^{-1}\right) \quad 1 \quad 0.250 .06 \quad 0$

K81Ac/ MAT Il $\alpha$ ratio $\quad \begin{array}{lllll}1.0 & 1.6 & 2.4 & 3.6\end{array}$

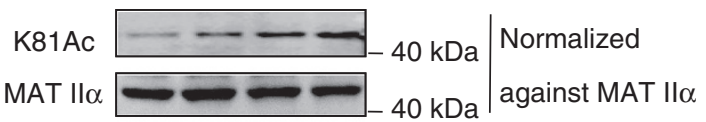

MAT II $\alpha / \beta$-actin ratio $\quad \begin{array}{llll}1.0 & 0.8 & 0.5 & 0.2\end{array}$

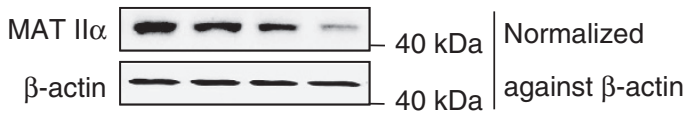

b

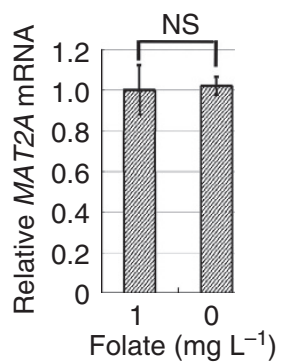

d

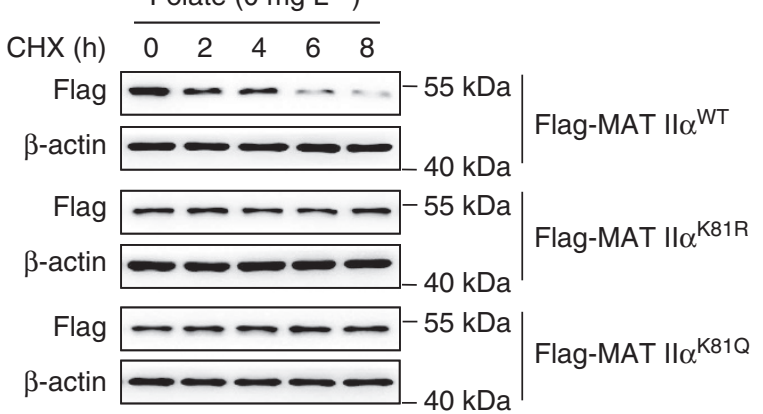

C

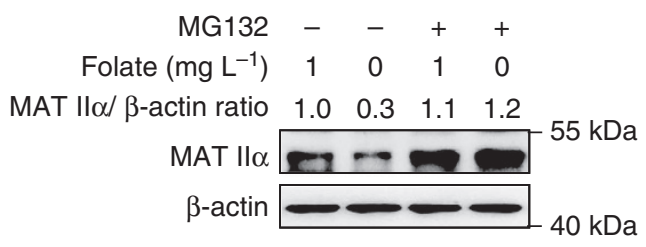

e

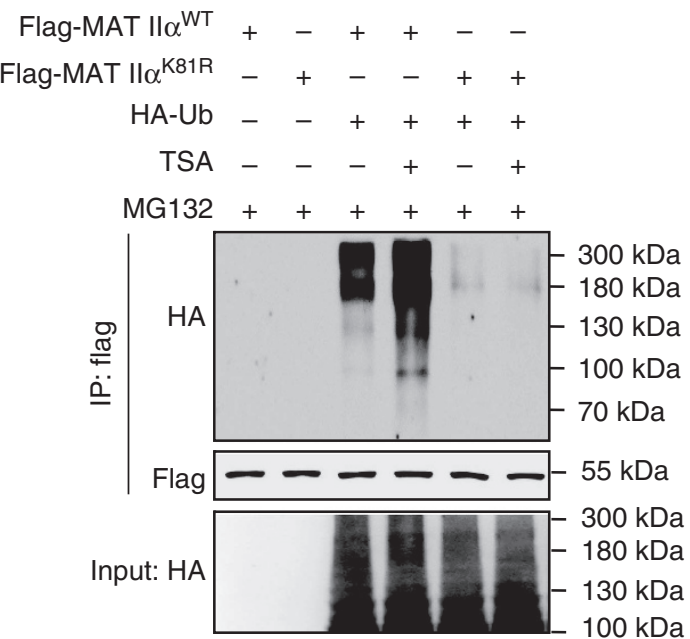

$\mathbf{f}$
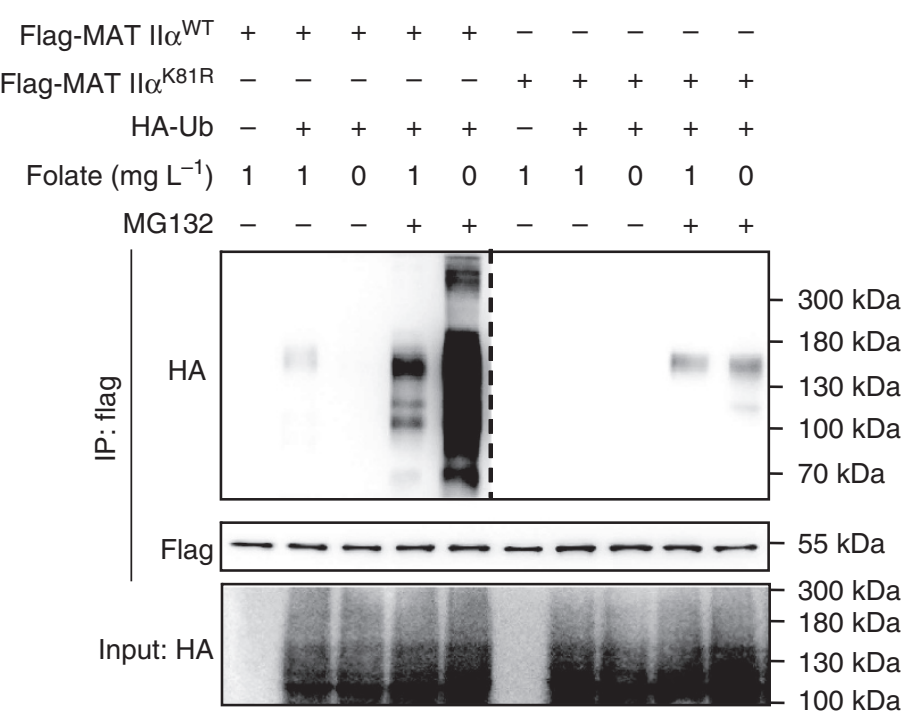

9

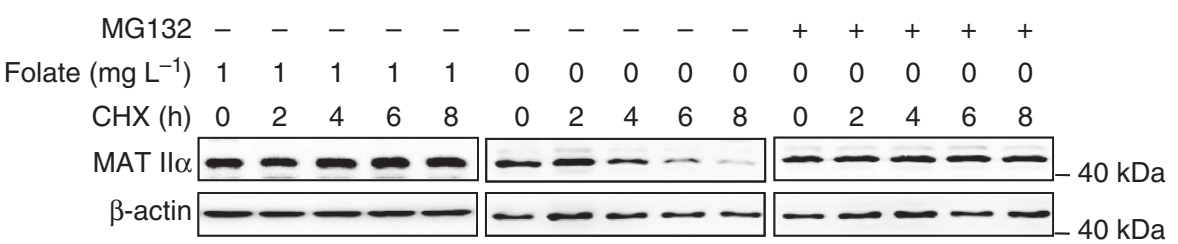

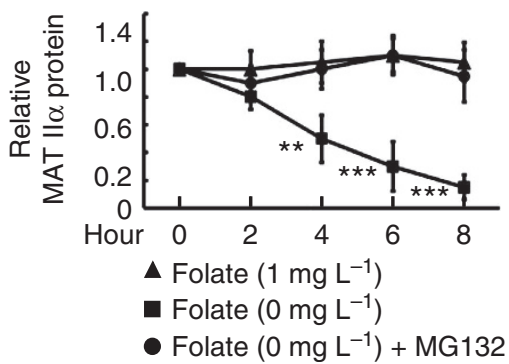


experiment showed that $U B R 4$ knockdown could totally block the decreased half-life of MAT II $\alpha$ upon folate deprivation (Fig. 3c). Moreover, UBR4 knockdown increases MAT II $\alpha$ protein level and its acetylation level (Supplementary Fig. 3, left panel). The efficacy of UBR4 knockdown was validated by quantitative PCR (qPCR; Supplementary Fig. 3, right panel). In addition, we performed ubiquitin ladder experiment to visualize ubiquitylation. Consistent with previous findings, polyubiquitylation signal of MAT II $\alpha$ was detected upon folate deprivation (Fig. 3d), whereas HAtagged ubiquitin could no longer be detected at high molecular weight after knocking down UBR4 expression (for quantification of UBR4 mRNA level, please refer to Fig. 3d). Collectively, these results verify UBR4 as an E3 ligase participating in polyubiquitylation-mediated proteasomal degradation of MAT II $\alpha$.

P300 acetylates MAT II $\alpha$ at K81. To identify the acetyl-transferase responsible for MAT II $\alpha$ K81 acetylation, we co-transfected four HATs (histone acetyl-transferases), P300 (E1A binding protein), PCAF ( $\mathrm{P} 300 / \mathrm{CBP}$-associated factor, also known as $\mathrm{K}$ (lysine) acetyl-transferase 2B, KAT2B), CBP (CREB-binding protein) and GCN5 (KAT2A), individually with MAT2A into HEK293T cells, and found P300 overexpression increased MAT II $\alpha$ acetylation, whereas others did not (Fig. 4a). In addition, overexpression of P300 increased endogenous K81 acetylation of MAT II $\alpha$ (Fig. $4 b$ ). To further verify whether P300 acetylates MAT II $\alpha$ at K81, we designed experiment shown in Supplementary Fig. 4, and proved that P300 overexpression only increases acetylation of wild-type MAT II $\alpha$, but not the K81R/Q mutant. Conversely, knocking down P300 by RNA interference significantly reduced K81 acetylation of endogenous MAT II $\alpha$ (Fig. 4c). P300 decreased MAT II $\alpha$ protein level in a dosedependent manner (Fig. 4d), whereas P300 knockdown rescued folate deprivation-induced MAT II $\alpha$ destabilization (Fig. 4e). Moreover, we found folate-deprivation increased the interaction between endogenous MAT II $\alpha$ and P300 (Fig. 4f), and this interaction was further verified by glutathione $S$-transferase (GST) pull-down (Fig. 5f). To further examine the hypothesis that P300 is the acetyl-transferase of MAT II $\alpha$, in vitro acetylation assay was conducted. As shown in Fig. 4g, recombinant P300 instantly acetylated MAT II $\alpha$ at K81. Collectively, our results demonstrate that $\mathrm{P} 300$ is the acetyl-transferase responsible for MAT II $\alpha$ acetylation at K81.

HDAC3 deacetylates MAT II $\alpha$ at K81. According to the previous results that TSA, an inhibitor of type I, II and IV HDACs, increased K81 acetylation of MAT II $\alpha$, we postulated that HDACs might be involved in MAT II $\alpha$ deacetylation. We co-expressed HDAC1-7 individually with MAT II $\alpha$, and found that overexpression of HDAC3, but not other tested HDACs, decreased the acetylation level of ectopically expressed MAT II $\alpha$ (Fig. 5a and Supplementary Fig. 5). Thereafter, we overexpressed HDAC3 in HEK293T cells, and found a decrease in K81 acetylation of endogenous MAT II $\alpha$ (Fig. 5b). Notably, HDAC3 knockdown decreased the steady protein level of MAT II $\alpha$ (Fig. 5c). Furthermore, we found HDAC 3 knockdown led to the destabilization of MAT II $\alpha$ (Fig. 5d), further supported that deacetylation stabilizes MAT II $\alpha$. Previous study by Katoh et al. identified HDAC3 as a putative MAT II $\alpha$-interacting protein by MS analysis ${ }^{19}$. To verify this interaction between HDAC3 and MAT II $\alpha$, we performed co-immunoprecipitation (co-IP) experiment and found that interaction indeed exists between these two proteins, and the interaction could be further enhanced in the presence of folate (Fig. 5e). Similarly, GST pull-down was performed to further verify this interaction (Fig. 5f). Taken together, HDAC3 is the deacetylase for MAT II $\alpha$ K81 acetylation.

\section{K81 mutants promote tumour growth in vitro and in vivo.} Given MAT II $\alpha$ overexpression was previously implicated to promote cancer cell proliferation and cancer progression ${ }^{7,15}$, we next examined the effect of MAT II $\alpha$ acetylation on cell proliferation and tumour growth. To this end, we generated stable cell lines in HepG2 cells in which endogenous MAT II $\alpha$ was knocked down by short hairpin RNA (shRNA; targeting $3^{\prime}$ untranslated region of $M A T 2 A$ gene) and the shRNA-resistant MAT II $\alpha$ wild-type, K81Q or K81R (each contains only coding sequence of wild-type or mutated MAT2A gene) was stably expressed. Western blotting analysis demonstrated that the endogenous MAT II $\alpha$ was effectively knocked down and that wild-type, K81Q or K81R mutants were expressed at levels similar to that of the endogenous MAT II $\alpha$ (Fig. 6a). Direct cell count and MTT assays were both applied to monitor cell proliferation velocities of wild-type or K81 mutant stable cells under normal or folate-deprived conditions. MAT2A knockdown instantly caused growth arrest in HepG2 stable cell line (Supplementary Fig. 6a). HepG2 cells expressing K81R and K81Q mutant stable cells were found proliferating significantly faster than cells expressing wildtype MAT II $\alpha$ upon folate deprivation, whereas under normal condition, no significant difference in proliferating velocity was detected (Fig. 6b and Supplementary Fig. 6b). As MAT II $\alpha$ catalyses the production of main methyl donor SAM, and SAM is converted to SAH (S-adenosyl-homocysteine) after the methyltransferation reaction, we then investigated SAM/SAH

Figure 2 | Folate decreases K81 acetylation and stabilizes MAT Il $\boldsymbol{\alpha}$. (a) Folate deprivation increases K81 acetylation of MAT Il $\boldsymbol{\alpha}$ and decreases its protein level. HEK293T cells were cultured in medium containing folate of different concentrations as indicated for $48 \mathrm{~h}$. Western blotting was used to determine MAT $\| \alpha$ protein and K81 acetylation levels. (b) Folate-deprivation has no effect on MAT2A mRNA level. MAT2A mRNA was determined by qPCR and normalized against $\beta$-actin. Error bars represent \pm s.d. of triplicate experiments. The two-tailed Student's $t$-test was used. NS denotes no significance. (c) MG132 rescues the decrease in MAT II $\alpha$ protein induced by folate deprivation. HEK293T cells were cultured as indicated and treated with either dimethylsulphoxide (DMSO; solvent) or $10 \mu \mathrm{M}$ MG132 for $6 \mathrm{~h}$. Cell lysates were analysed by western blotting. (d) Folate deprivation exclusively shortens the half-life of wild-type MAT $\| \alpha$, but not K81R or K81Q mutant. HEK293 stable cell lines expressing wild-type MAT Il $\alpha$, or K81R/K81Q mutants were cultured in folate-deprived condition for $48 \mathrm{~h}, \mathrm{CHX}\left(10 \mu \mathrm{g} \mathrm{ml}{ }^{-1}\right)$ treatment was applied for different time course before harvest. MAT Il $\alpha$ protein levels were determined by western blotting (left panel). The right panel showcases relative protein amounts of different groups. Error bars represent \pm s.d. of triplicate experiments. The two-tailed Student's $t$-test was used. ${ }^{\star \star} P<0.01$; ${ }^{\star \star \star} P<0.001$. (e) K81R mutation blocks MAT Il $\alpha$ ubiquitylation induced by TSA. HEK293T cells were transfected with the indicated plasmids. After TSA and MG132 treatment as indicated, ubiquitylation of purified flag-MAT Il $\alpha$ proteins were determined. (f) Folate deprivation promotes ubiquitylation of wild-type MAT Il $\alpha$ but not K81R mutant. HEK293T cells were transfected as indicated. Ubiquitylation of flag-MAT Il $\alpha$ protein was determined by western blotting. (g) MG132 stabilizes MAT Il $\alpha$ upon folate deprivation. HEK293T cells were cultured under normal or folate-deprived condition, followed by CHX and MG132 treatment. Cell lysates were directly subjected to western blotting (left panel). The right panel showcases relative protein amounts of different groups. Error bars represent \pm s.d. of triplicate experiments. The two-tailed

Student's $t$-test was used. ${ }^{\star \star} P<0.01 ;{ }^{\star \star \star} P<0.001$. 
a

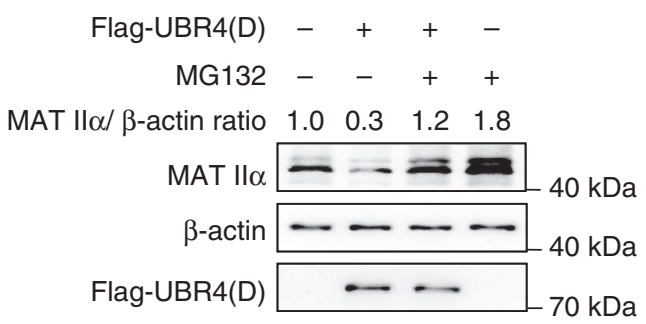

C

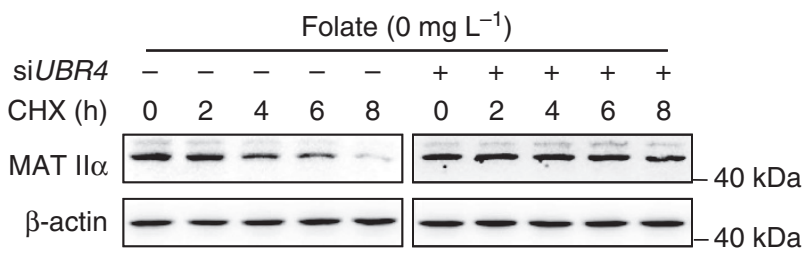

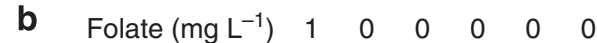

siUBR4 - $-1 \quad \begin{array}{llll}1 & 2 & 3\end{array}$

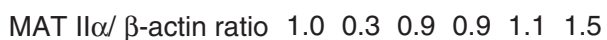
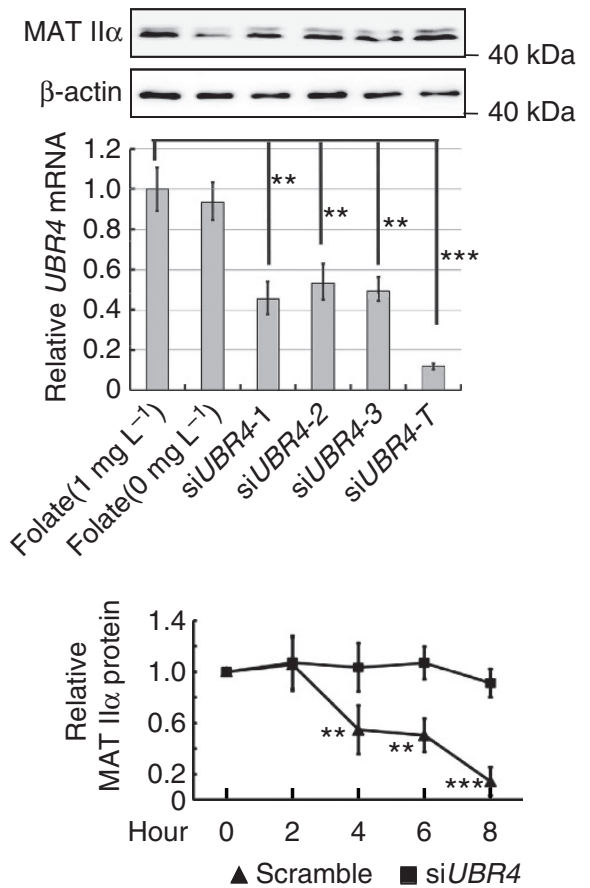

d
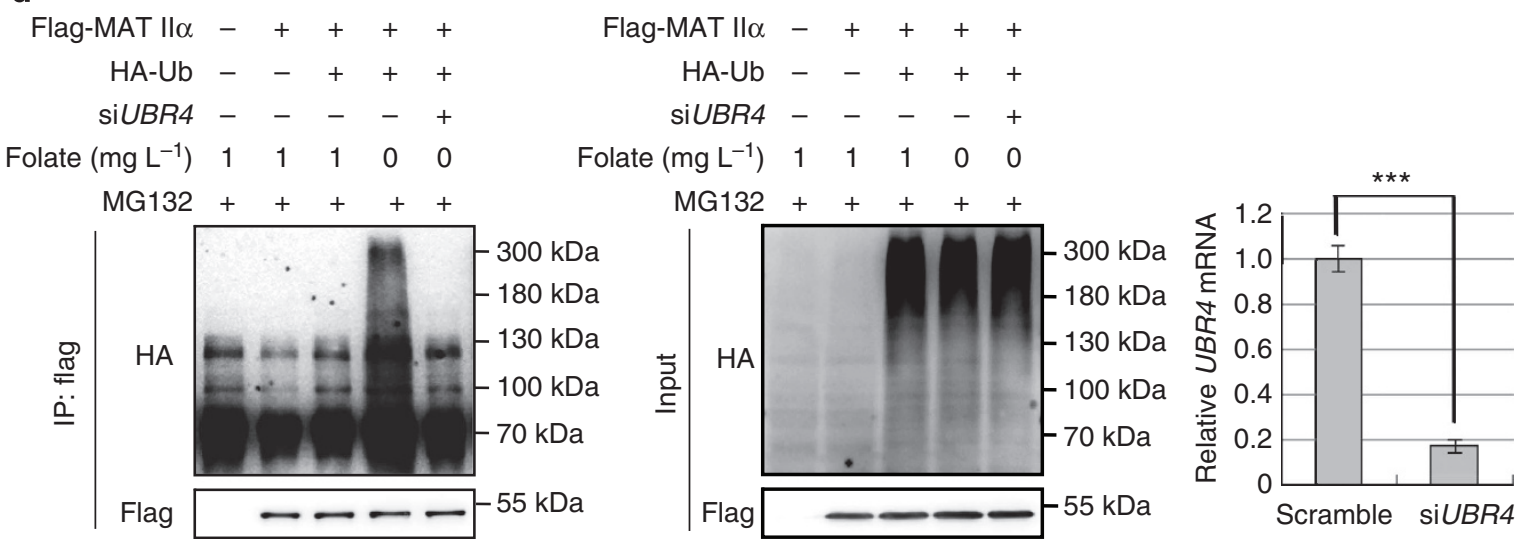

Figure 3 | UBR4 targets MAT Il $\boldsymbol{\alpha}$ for degradation. (a) UBR4 overexpression decreases MAT Il $\alpha$ protein. HEK293T cells were transfected as indicated (UBR4 (D) represents a truncated form of UBR4 protein, with substrate-binding and catalytic domains only), followed by MG132 treatment. Cell lysates were directly subjected to western blotting. (b) UBR4 knockdown rescues MAT Il $\alpha$ protein reduced by folate deprivation. Three different siRNA oligos siUBR4-1, -2 and -3 were transfected, respectively, or together (siUBR4-T) into HEK293T cells. Protein levels of MAT Il $\alpha$ were determined by western blotting. UBR4 knockdown efficiency was analysed by qPCR. Values were normalized against $\beta$-actin and compared with the relative mRNA of folate (1 mg $\mathrm{I}^{-}$ ${ }^{1}$ ) group (set as 1.0). Error bars represent \pm s.d. of triplicate experiments. The two-tailed Student's $t$-test was used. ${ }^{\star \star} P<0.01$; ${ }^{\star \star \star} P<0.001$. (c) UBR4 knockdown increases MAT Il $\alpha$ stability. HEK293T cells were transfected with siUBR4 or control. CHX chase experiment was performed and MAT Il $\alpha$ protein was determined by western blotting (left panel). The right panel showcases relative protein amounts of different groups. Error bars represent \pm s.d. of triplicate experiments. The two-tailed Student's $t$-test was used. ${ }^{\star \star} P<0.01$; ${ }^{\star \star \star} P<0.001$. (d) UBR4 knockdown blocks folate-deprivation inducedubiquitylation of MAT Il $\alpha$. HEK293T cells were transfected as indicated and cultured in folate containing or deprived culture medium. Ubiquitylation assay was conducted. The efficiency of UBR4 knockdown was validated by qPCR. Error bars represent \pm s.d. of triplicate experiments. The two-tailed Student's $t$-test was used. ${ }^{\star \star \star} P<0.001$.

ratio in different stable cell lines and found that folate deprivation significantly decreased SAM/SAH ratio in wild-type but not in K81R/Q mutant-expressing cells (Supplementary Fig. 6c). As a result, global methylation in wild-type-expressing cells cultured upon folate deprivation was found decreased, compared with that of the mutant-expressing cells grew under the same condition (Supplementary Fig. 6d).
To determine folate's effect on MAT II $\alpha$ K81 acetylation in tumour growth, we performed xenograft experiments using HepG2 stable cell lines referred previously. Nude mice were subcutaneously injected with HepG2 stable cells and fed with folate-free diet. Tumour growth was continuously monitored thereafter. Measurement of the widths and lengths of tumours demonstrated that HepG2 cells expressing K81R or K81Q 
a

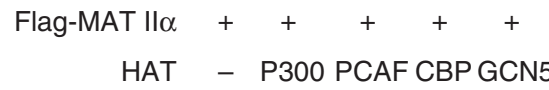

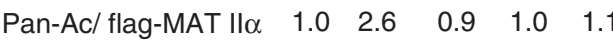

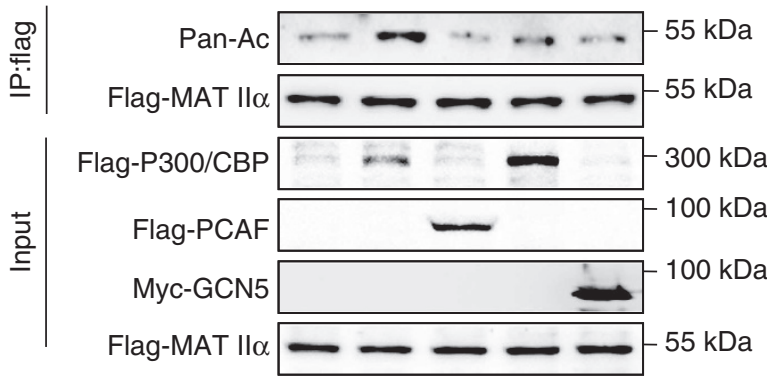

b

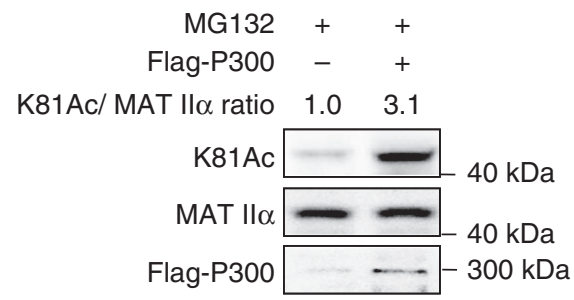

C SiP300 - +

$\mathrm{K} 81 \mathrm{Ac} / \mathrm{MAT}$ Il $\alpha$ ratio $\quad 1.0 \quad 0.2$

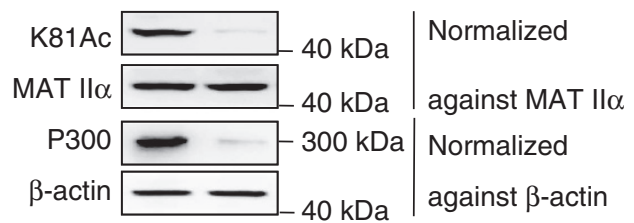

e d

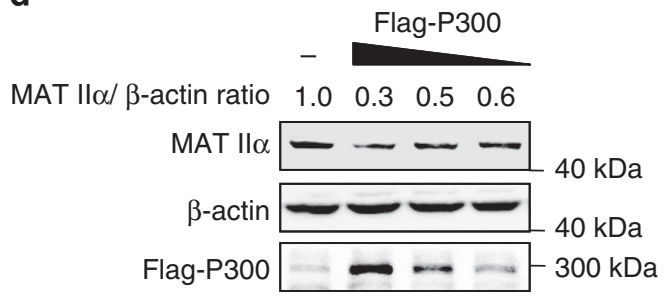

\begin{tabular}{|c|c|c|c|c|c|c|c|c|c|c|}
\hline siP300 & - & - & - & - & - & + & + & + & + & + \\
\hline $\mathrm{CHX}(\mathrm{h})$ & 0 & 2 & 4 & 6 & 8 & 0 & 2 & 4 & 6 & 8 \\
\hline MAT II $\alpha$ & $=$ & - & - & - & - & - & - & - & - & - \\
\hline$\beta$-actin & & - & - & & - & & & & & \\
\hline
\end{tabular}

f

g
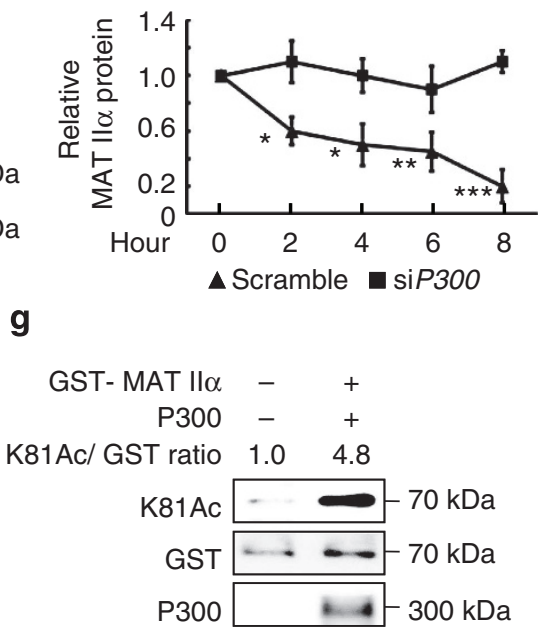

Figure 4 | P300 acetylates MAT II $\boldsymbol{\alpha}$. (a) Overexpression of P300, not other HATs, increases acetylation of exogenous MAT II $\alpha$. HEK293T cells were transfected as indicated and acetylation of flag-MAT Il $\alpha$ was determined using Pan-Ac antibody. (b) Overexpression of P300 increases K81 acetylation of endogenous MAT $\| \alpha$. HEK293T cells were transfected with flag-P300 and K81 acetylation of MAT Il $\alpha$ was determined using K81Ac antibody. (c) P300 knockdown decreases MAT II $\alpha$ K81 acetylation. HEK293T cells were transfected with siP300 or control and MAT II $\alpha$ acetylation levels were determined by western blotting. (d) P300 decreases protein levels of MAT Il $\alpha$. Flag-P300 was transfected in declined doses into HEK293T cells and cell lysates were measured by western blotting. (e) P300 knockdown increases MAT Il $\alpha$ stability. HEK293T cells were transfected with siP300 or control and cultured under folate-deprived condition before $\mathrm{CHX}$ was added and treated for indicated durations. Levels of endogenous MAT Il $\alpha$ protein were determined by western blotting and normalized against $\beta$-actin (left panel). The right panel showcases relative protein amounts of different groups. Error bars represent \pm s.d. of triplicate experiments. The two-tailed Student's $t$-test was used. ${ }^{\star} P<0.05$; ${ }^{\star \star} P<0.01 ;{ }^{\star \star \star} P<0.001$. (f) Folate-deprivation enhances MAT $\| \alpha-P 300$ interaction. HEK293T cells were transfected with flag-tagged MAT Il $\alpha$ and cultured in normal or folate-deprived conditions. The interaction between flagMAT Il $\alpha$ and endogenous P300 was determined by co-IP followed by western blotting. (g) P300 acetylates MAT Il $\alpha$ at K81 in vitro. Ectopically expressed GST-MAT Il $\alpha$ from BL21 E. coli was purified through GST antibody-conjugated beads and incubated with P300 (immunoprecipitation-purified and eluted). K81 acetylation levels were analysed using K81Ac antibody and normalized against GST-MAT Il $\alpha$.

mutants displayed a growth rate significantly faster than those expressing wild-type MAT II $\alpha$ upon folate-deprived dietary (Fig. 6c and Supplementary Fig. 6e). At the time of harvesting, mice injected with cells expressing K81R/K81Q mutants developed larger tumours than those injected with cells expressing wild-type MAT II $\alpha$ (Fig. 6d). In addition, levels of MAT II $\alpha$ protein in stable cell lines and xenograft tumours derived from stable cell lines were assessed by western blotting, protein levels of K81R and K81Q mutants were found indeed higher than that of the wild-type MAT II $\alpha$ (Fig. 6e). Collectively, K81 mutants stabilize MAT II $\alpha$ and promote tumour cell growth upon folatedeprivation both in vitro and in vivo. 
a

$\begin{array}{rrrrrrrrr}\text { Flag-MAT Il } \alpha & + & + & + & + & + & + & + & + \\ \text { HA-HDAC } & 1 & 2 & 3 & 4 & 5 & 6 & 7 & -\end{array}$

$\begin{array}{lllllllll}\text { Pan-Ac/ flag ratio } & 1.0 & 1.1 & 0.2 & 0.8 & 1.1 & 1.2 & 1.1 & 1.2\end{array}$

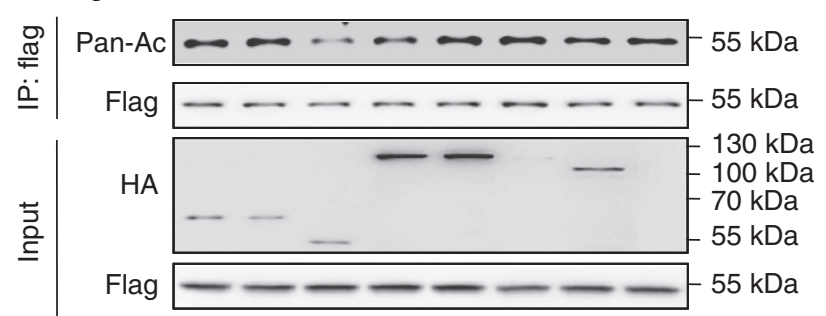

b

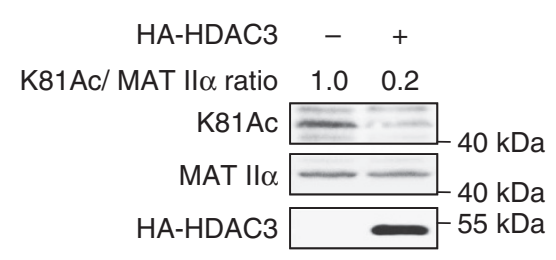

c

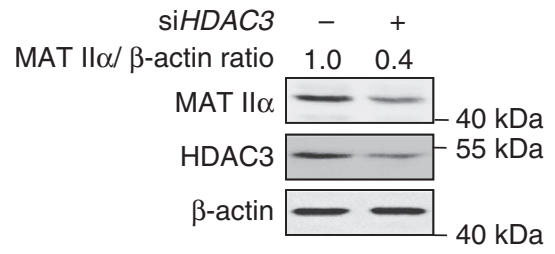

e d
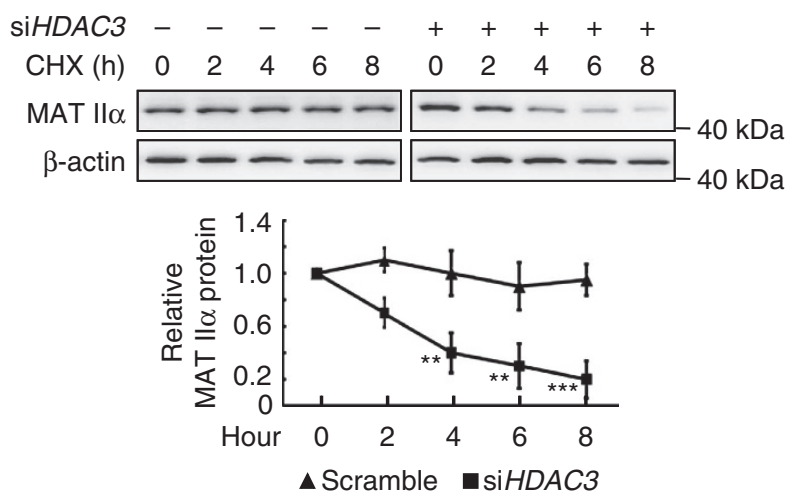

f

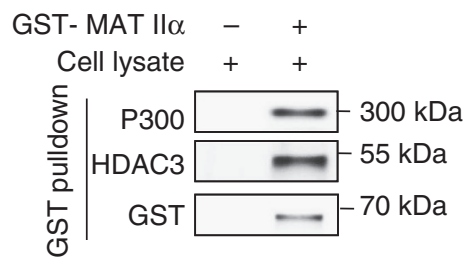

Figure 5 | HDAC3 deacetylates MAT Il $\boldsymbol{\alpha}$. (a) Overexpression of HDAC3, but not other HDACs, decreases the acetylation level of MAT II $\boldsymbol{\alpha}$. Each of the HA-tagged HDAC1-7 was co-transfected with flag-tagged MAT Il $\alpha$ into HEK293T cells and the acetylation levels of MAT Il $\alpha$ were determined by standard western blotting. (b) HDAC3 decreases K81 acetylation of endogenous MAT Il $\alpha$. HEK293T cells were transfected with HA-HDAC3. MAT II $\alpha$ K81 acetylation was detected using K81Ac antibody. (c) HDAC3 knockdown decreases MAT Il $\alpha$ protein. HEK293T cells were transfected with siHDAC3 or control. Cells were harvested $48 \mathrm{~h}$ after transfection, and lysates were analysed by western blotting. (d) HDAC3 knockdown destabilizes MAT Il $\alpha$. HEK293T cells were transfected with siHDAC3 or control, respectively. $\mathrm{CHX}$ chase treatment was applied as indicated. Cell lysates were directly subjected to western blotting (upper panel) and normalized against $\beta$-actin. The lower panel showcases relative protein amounts of different groups. Error bars represent \pm s.d. of triplicate experiments. The two-tailed Student's $t$-test was used. ${ }^{\star \star} P<0.01 ;{ }^{\star \star \star} P<0.001$. (e) Folate increases the interaction between HDAC3 and MAT Il $\alpha$. HEK293T cells were cultured with or without folate for $48 \mathrm{~h}$ and treated with MG132 for $6 \mathrm{~h}$ before harvest. Interaction between endogenous MAT Il $\alpha$ and HDAC3 was determined by co-IP and western blotting. (f) GST-MAT Il $\alpha$ can readily pull-down P300 and HDAC3. BL21 E. coli transformed with pGEX-GSTMAT II $\alpha$ plasmid was induced (or not induced) by isopropyl- $\beta$-D-thiogalactoside. Protein was then purified through GST antibody-conjugated column and incubated with HEK293T cell lysates (lysed by 0.3\% Nonidet P40 buffer) before re-purified through immunoprecipitation and subjected to western blotting.

K81 acetylation is decreased and inversely correlated with MAT II $\alpha$ in human hepatocellular cancer. MAT II $\alpha$ overexpression is implicated in human hepatocellular carcinoma (HCC). The finding that K81Q or K81R mutant promotes HepG2 cell proliferation prompted us to examine K81 acetylation in human hepatocellular cancers. We collected 80 pairs of primary human hepatocellular cancer samples with adjacent normal tissues for direct immunoblotting. Results showed that, compared with the matched normal liver tissues, 40 pairs of samples showed a significant increase of total MAT II $\alpha$ protein in cancerous tissues (Fig. 6f and Supplementary Fig. 6f). To further exam the correlation between K81 acetylation and MAT II $\alpha$ protein, we determined MAT II $\alpha$ K81 acetylation in these 40 pairs of samples, and found that 32 pairs of samples showed relatively higher levels of K81 acetylation in the tumour tissues than that of the matched normal tissues (Fig. $6 f$ and Supplementary Fig. 6g). Furthermore, we found that HDAC3 expression was increased in 19 out 32 HCC samples (Supplementary Fig. 6f). The tumour sample analyses demonstrate that MAT II $\alpha$ protein levels are elevated in hepatocellular cancers, and K81 acetylation inversely correlates with the elevated MAT II $\alpha$ protein. These data also indicate an application prospect of using MAT II $\alpha$ and K81 acetylation as potential biomarkers for hepatocellular cancer diagnosis. 
a

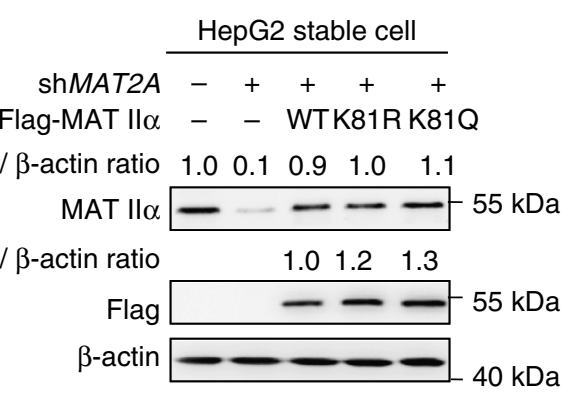

b

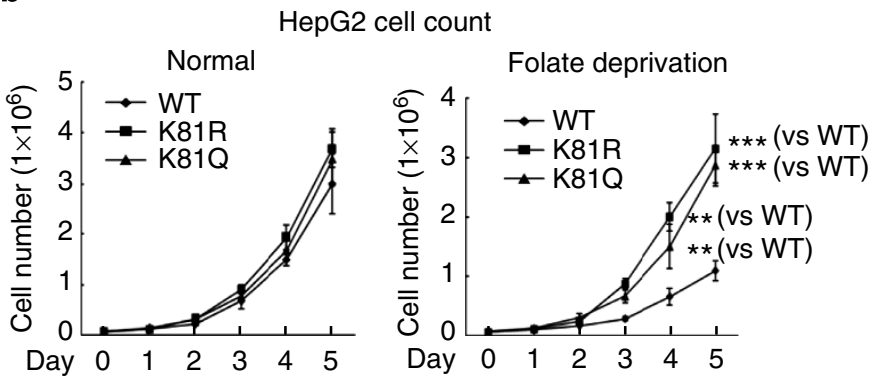

c

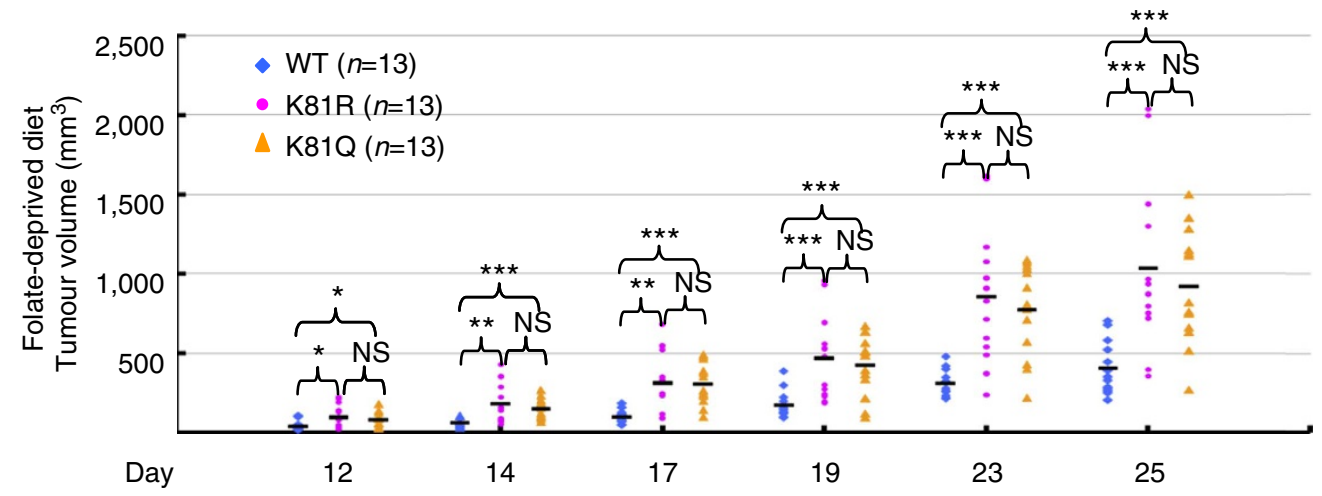

d
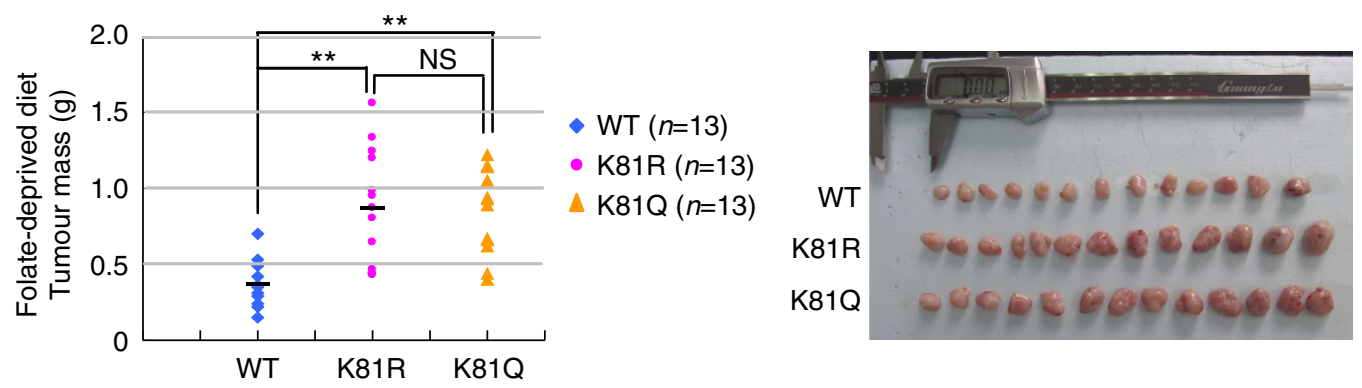

e

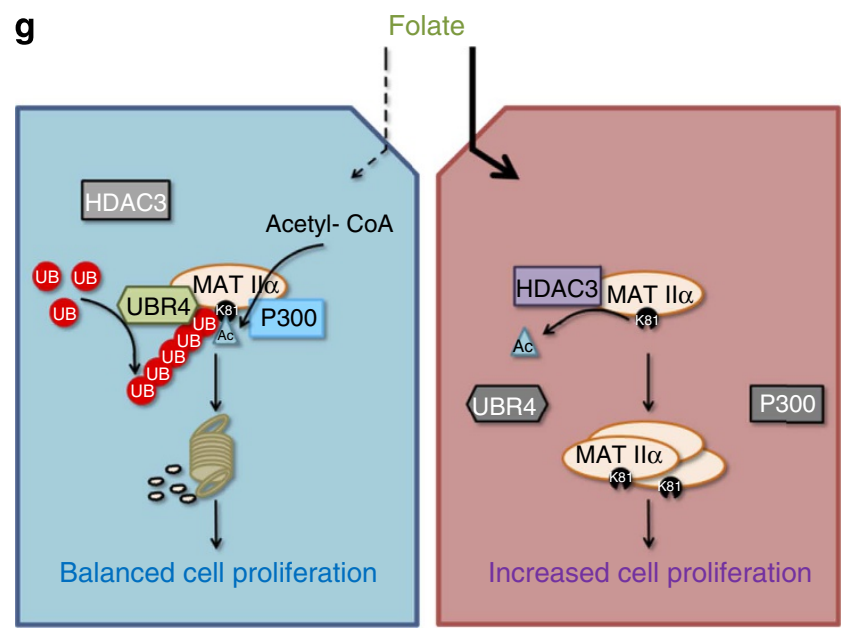

$\operatorname{shMAT2A} \frac{\text { HepG2 stable cell }}{-+++++\frac{\text { Xenograft }}{+++}}$

Flag-MAT Il $\alpha-\quad-$ WT K81R K81Q WT K81R K81Q MAT $\| \alpha / \beta$-actin ratio $\begin{array}{lllllllll}1.0 & 0.1 & 0.8 & 1.1 & 1.1 & 1.6 & 1.1 & 1.2\end{array}$

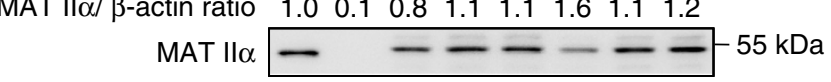

Flag/ $\beta$-actin ratio $\begin{array}{llllll}1.0 & 1.3 & 1.3 & 0.7 & 1.1 & 1.2\end{array}$

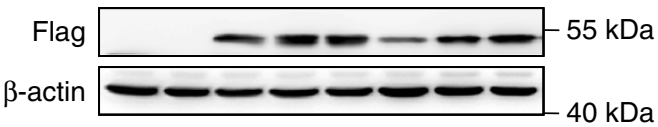

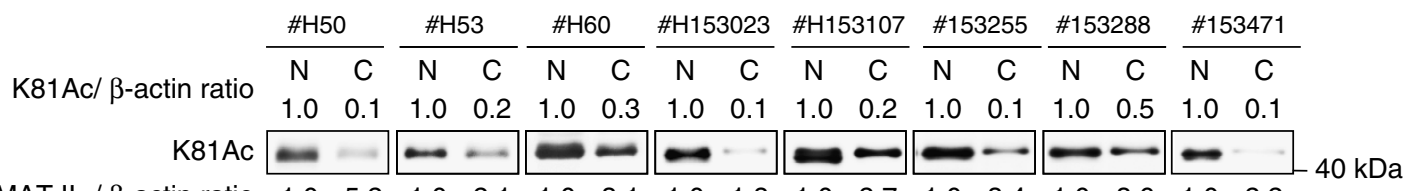

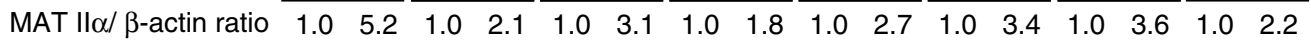

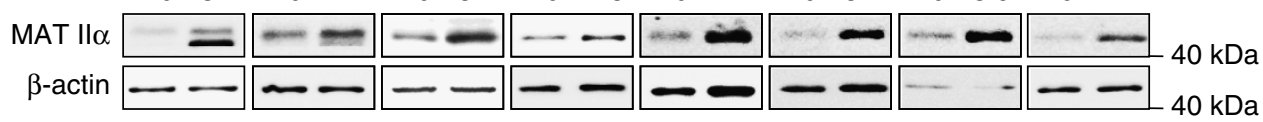




\section{Discussion}

Dysregulation of cellular metabolism is a hallmark of cancer. Besides elevated glycolysis, increased lipogenesis and so on, dysregulation of amino-acid metabolism is also implicated in cancer. Imbalanced methionine metabolism in human liver cancer has been known for at least 60 years. Liver can be considered as the body's SAM factory, and MAT is the key enzyme catalysing biosynthesis of SAM. Overexpression of $M A T 2 A$ is common in various types of cancer ${ }^{6,7,15}$, whereas knocking down MAT2A gene expression causes cell cycle arrest and apoptosis in cancer cells ${ }^{10,11}$. Our study reveals a novel mechanism of MAT II $\alpha$ regulation by acetylation and ubiquitylation in response to folate concentration (Fig. 6g) and provides a new mechanistic insight in MAT II $\alpha$ upregulation in cancer.

Upregulation of MAT II $\alpha$ in cancer is in part due to MAT2A overexpression regulated by alerted gene transcription or mRNA stability ${ }^{13-15}$. In this study, we report two post-translational modifications of MAT II $\alpha$. We propose that MAT II $\alpha$ acetylation modulated by folate concentration and consequent ubiquitylation play crucial roles in MAT II $\alpha$ stability control. When folate is sufficient, MAT II $\alpha$ is stabilized via HDAC3 deacetylation and subsequent inhibition of ubiquitylation. Accumulation of MAT II $\alpha$ therefore enhances tumour growth. Conversely, when folate is deprived, P300 acetylates MAT II $\alpha$ at K81. Acetylation therefore promotes UBR4-mediated MAT II $\alpha$ ubiquitylation, leading to its proteasomal degradation and reduced cell growth.

Folate is needed in large quantities in rapidly proliferating cells. Folate uptake is increased in several types of solid human cancers including ovarian, endometrial, colorectal, kidney, lung and breast carcinomas ${ }^{20-22}$. Anti-folate drugs have long been approved for treating cancers ${ }^{23}$. Therefore, one may expect an important role for folate in MAT II $\alpha$ regulation.

Indeed, folate-induced MAT II $\alpha$ stabilization results in an increase in SAM production (Supplementary Fig. 6c). We speculate that SAM accumulation would possibly cause dysregulations of oncogenes and tumour-suppressor genes via affecting global methylation, eventually leading to tumorigenesis and cancer development. In addition, the fact that stabilization of MAT II $\alpha$ by K81 mutations significantly promotes cancer cell proliferation and in vivo tumour growth indicates a critical role of MAT II $\alpha$ acetylation in coordinating folate availability and the regulation of cell growth and tumorigenesis. It also suggests a possibility that promoting MAT II $\alpha$ acetylation may merit exploration as a target for cancer therapy. Notably, the observation that MAT II $\alpha$ is overexpressed in hepatocellular cancer tissues, whereas K81 acetylation is significantly decreased indicates a pivotal role of MAT II $\alpha$ acetylation regulation in hepatocellular cancer development.

\section{Methods}

Cell culture and treatment. Cells were all purchased from American Type Culture Collection and cultured in DMEM/high-glucose medium (HyClone) supplemented with $10 \%$ fetal bovine serum (HyClone), $1 \%$ penicillin and streptomycin at $37^{\circ} \mathrm{C}$, in a humidified atmosphere containing $5 \% \mathrm{CO}_{2}$. Standard RPMI- 1640 and RPMI-1640 without folate were purchased from Life Technologies, Inc. (GIBCO). For folate-deprivation treatment, cells were cultured in the medium containing different amounts of folate as indicated. For TSA, MG132, CHX treatment, please see descriptions in figure legends, respectively.

Immunoprecipitation and western blotting. Cells were lysed in $0.3 \%$ Nonidet P40 buffer ( $150 \mathrm{mM} \mathrm{NaCl}, 50 \mathrm{mM}$ Tris- $\mathrm{HCl}, \mathrm{pH} 7.5)$ containing inhibitors (1 mM phenylmethylsulphonyl fluoride, $1 \mu \mathrm{g} \mathrm{ml}^{-1}$ of aprotinin, $1 \mu \mathrm{g} \mathrm{ml}^{-1}$ of leupeptin, $1 \mu \mathrm{g} \mathrm{ml}^{-1}$ of pepstatin, $1 \mathrm{mM} \mathrm{Na}_{3} \mathrm{VO}_{4}, 1 \mathrm{mM} \mathrm{NaF}, 30 \mu \mathrm{M}$ TSA and $15 \mathrm{mM}$ NAM, all in their final concentrations). Cell debris were removed by centrifuging at $4{ }^{\circ} \mathrm{C}$, 13,000 r.p.m. for $15 \mathrm{~min}$, and lysates were incubated for $3 \mathrm{~h}$ at $4{ }^{\circ} \mathrm{C}$ with anti-flag M2 agarose (Sigma). The immunoprecipitates were washed three times with $0.3 \%$ Nonidet P40 buffer before boiled and analysed by western blotting according to the standard methods. The following primary antibodies were commercially obtained: Flag (Sigma, with 1:10,000 working dilution), HA (Santa Cruz, with 1:1,000 working dilution), $\beta$-actin (Sigma, with 1:10,000 working dilution), P300 (Santa Cruz, with 1:1,000 working dilution), MAT II $\alpha$ (Santa Cruz, with 1:1,000 working dilution), acetylated-lysine (Cell Signaling, with 1:1,000 working dilution). Antibody specific to MAT II $\alpha$ K81Ac was prepared commercially from immunizing rabbits at Shanghai Genomic Inc. (with 1:2,000 working dilution). Images from western blotting have been cropped for presentation. Full-size images are presented in Supplementary Fig. 7.

Ubiquitin ladder assay. Ubiquitin ladder assay was performed as previously described ${ }^{24}$. Thirty-six hours after transfection, cells were collected and lysed in $1 \%$ SDS buffer (50 mM Tris- $\mathrm{HCl}$ (pH 7.5), $0.5 \mathrm{mM}$ EDTA, $1 \mathrm{mM}$ dithiothreitol) with inhibitors and boiled for $10 \mathrm{~min}$. Before immunoprecipitation, lysates were diluted ten-fold with $0.3 \%$ Nonidet P40 buffer. Ubiquitylation were determined by western blotting.

siRNA transfection and RNA interference. Downregulation of UBR4, P300 and HDAC3 was performed by RNA interference. Synthetic siRNA oligonucleotides were obtained commercially from Shanghai Genepharma Co, Ltd. Sequences of effective sequences are as follow:

siUBR4-1: 5'-CGUCCCAGAAUGCCUUAAAdTdT-3'

siUBR4-2: 5'-GCUGGUAGUUAUGGUGAAAdTdT-3'

siUBR4-3: 5'-GGACCAUCAACCUGUAUUAdTdT- $3^{\prime}$

siP300-1: 5'-UGACACAGGCAGGCUUGACdTdT-3'

siP300-2: 5'-AACAGAGCAGUCCUGGAUUAGdTdT-3'

siHDAC3: 5'-CCGCCAGACAAUCUUUGAAdTdT- $3^{\prime}$

All siRNA transfections were performed as described in Lipofectamine 2000 (Invitrogen) standard protocol. The knockdown efficiency was verified by western blotting or qPCR.

Knocking down and putting back stable cell lines. Flag-tagged human wild type, K81R and K81Q mutants of MAT2A were cloned into the retroviral vector (pQCXIH) and were co-transfected into HEK293T cells together with vectors expressing gag and $v s v g$ genes (from vesicular stomatitis virus G). Retroviral supernatants were harvested by filtration $(0.45 \mu \mathrm{m}$ filters $) 48 \mathrm{~h}$ after initial plasmid transfection and mixed with polybrene $\left(8 \mu \mathrm{g} \mathrm{ml}^{-1}\right.$ as final concentration) to increase the infection efficiency before applied to HepG2 cells. HepG2 cells were infected with the prepared virus for $12 \mathrm{~h}$ and screened by hygromycin $\left(350 \mu \mathrm{g} \mathrm{ml}^{-1}\right.$ as final concentration) for at least 2 weeks. pMKO-shMAT2A and pMKO-shVEC were

Figure 6 | K81 mutations promote tumour cell growth in vitro and in vivo. (a) Verification of HepG2 stable cell lines. Knockdown efficiency and reexpression levels of wild-type or K81R/Q mutants were determined by western blotting. (b) K81R and K81Q mutations reverse the proliferative disadvantage of HepG2 cells upon folate deprivation. HepG2 stable cell characterized in a were cultured in normal or folate-deprived medium. Direct cell count was performed every $24 \mathrm{~h}$ after seeding. Error bars represent cell numbers \pm s.d. for triplicate experiments. The two-tailed Student's $t$-test was used. ${ }^{\star \star} P<0.01 ;{ }^{\star \star \star} P<0.001$. (c,d) K81R and K81Q mutants promote xenograft tumour growth. Subcutaneous xenograft experiment was performed in nude mice using HepG2 stable cells. Major and minor diameters of tumours were measured and tumour volumes were calculated. The two-tailed Student's $t$-test was used. ${ }^{\star} P<0.05 ;{ }^{\star \star} P<0.01 ;{ }^{\star \star \star} P<0.001$; NS denotes no significance (c). 25 days after injection, tumours were dissected, photographed and weighted. The two-tailed Student's $t$-test was used. ${ }^{\star \star} P<0.01$; NS denotes no significance (d). (e) The expression of wild-type, K81R and K81Q MAT Il $\alpha$ in xenografts. Whole-cell lysates were prepared from either original HepG2 stable cell lines or xenograft tumours, followed by western blotting analysis. (f) The hepatocellular cancer clinical samples show an inverse correlation between MAT Il $\alpha$ protein and K81 acetylation. Human hepatocellular cancer samples each paired with cancerous tissue (designated as C) and adjacent normal tissue (designated as N) were lysed and directly subjected to western blotting. Only eight pairs of samples showcasing inverse correlation are shown. For more samples, please refer to supplementary Fig. 6f. (g) Working model. By enhancing MAT $\| \alpha$ association with P300 and UBR4, folate deprivation promotes both MAT $\| \alpha$ K81 acetylation and ubiquitylation, resulting in its proteasomal degradation. Folate stabilizes MAT Il $\alpha$ by dissociating MAT I $\alpha$-P300-UBR4 complex, and promoting MAT Il $\alpha$-HDAC3 interaction. Accumulated MAT II $\alpha$ protein facilitates rapid cell proliferation and, therefore, promotes tumour growth. 
constructed as Short Hairpin RNA vector. shRNA constructs including shMAT2A employed two effective sequences targeting $3^{\prime}$-untranslated region as follows: 5'-GCATAGGTGATCCATGTAACT-3'; 5' -GTAAGTTGGGCTTGCTATTCT-3' HepG2 stable cells expressing flag-tagged wild type, K81R or K81Q of MAT II $\alpha$ were infected with pMKO-shMAT2A and pMKO-sh VEC retrovirus. MAT II $\alpha$ knockdown efficiency of positive clones was determined after 2 weeks of drug selection (puromycin, $5 \mu \mathrm{g} \mathrm{ml}^{-1}$ as final concentration).

GST pull-down assay. BL21 E. coli transformed with pGEX-GST-MAT II $\alpha$ plasmid was induced (or not induced) by isopropyl- $\beta$-D-thiogalactoside $(0.1 \mathrm{mM}$ as final concentration) at $20^{\circ} \mathrm{C}$ for $12 \mathrm{~h}$. Protein was then purified through GST antibody-conjugated beads. Beads were then added to HEK293T cell lysate (lysed by $0.3 \%$ Nonidet P 40 buffer), and mixed at $4{ }^{\circ} \mathrm{C}$ overnight. Non-induced group was used as a negative control. Beads were subsequently harvested through centrifugation and washed four times by $0.3 \%$ Nonidet P 40 buffer before boiled by $1 \times$ SDS-polyacrylamide gel electrophoresis loading buffer and subjected to western blotting.

In vitro acetylation assay. Ectopically expressed MAT II $\alpha$ from BL21 E. coli was purified through GST antibody-conjugated beads and incubated with P300 (immunoprecipitation-purified and eluted) in histone acetyl-transferase (HAT) assay buffer (purchased from Millipore), supplemented with acetyl-co-enzyme A $\left(10 \mu \mathrm{M}\right.$ as final concentration). Reaction was conducted under $30^{\circ} \mathrm{C}$ for $1 \mathrm{~h}$ with continuous shaking. After adding $5 \times$ SDS-polyacrylamide gel electrophoresis loading buffer and boiled, samples were analysed by western blotting.

Cell proliferation analysis. $5 \times 10^{4} \mathrm{HepG} 2$ stable cells were seeded in triplicate in each well of six-well plate, and cultured in normal or folate-deprived medium. Cell numbers were counted every day over a 5 -day period via haemacytometer counting method. For MTT (3-(4,5-dimethyl-2-thiazolyl-2,5-diphenyl-2-H-tetrazolium bromide)) assays, $2 \times 10^{3} \mathrm{HepG} 2$ stable cells were seeded in quintuplicate in each well of 96-well plate. Standard methods were performed to track the cell proliferation.

Xenograft analysis. The procedures related to animal studies were approved by the Ethics Committee of the Institutes of Biomedical Sciences, Fudan University. Rodent diet without folate was purchased from Research Diets, Inc. Nude mice (nu/nu, six-week-old males) were fed with folate-deprived diet for 1 week, followed by injected subcutaneously with $5 \times 10^{6} \mathrm{HepG} 2$ stable cells. The mice were subsequently fed with folate-deprived diet. Major and minor diameters of tumours were measured every 2 days. Mice were killed after 25 days and tumours were collected and weighed.

Hepatocarcinoma cancer samples. Hepatocarcinoma cancer samples were obtained from the 10th People's Hospital, Shanghai (Tongji University Affiliated), with written consents from all investigated patients. The procedures related to human subjects were approved by the Ethics Committee of the Institutes of Biomedical Sciences, Fudan University. Direct immunoblotting was performed as mentioned above.

SAM and SAH quantification. SAM and SAH quantification were performed as previously described ${ }^{25}$. Cells were harvested and weighed. The cell pellets were added with $0.4 \mathrm{M}$ perchloric acid, mixed vigorously and centrifuged. Supernatants were adjusted to $\mathrm{pH}$ 5-7 with $2.5 \mathrm{M} \mathrm{K}_{2} \mathrm{HPO}_{4}$ and kept on ice for $15 \mathrm{~min}$ to precipitate potassium perchlorate. Samples were centrifuged twice and supernatants were analysed by liquid chromatography-tandem mass spectrometry.

Methylated DNA quantification. Genomic DNA was prepared using a DNA isolation kit (Beyotime). A methylated DNA quantification kit (Colorimetric; Abnova) was used to detect methylated DNA.

Statistic analysis. Two-tailed Student's $t$-tests were used for all comparisons, including $\mathrm{qPCR}$ analysis. All values included in the figures represent mean \pm s.d. Error bars represent \pm s.d. for triplicate experiments. The statistical significance is indicated as asterisks $\left(^{*}\right)$. Two-sided $P$ value of $<0.05$ was considered to be statistically significant $\left({ }^{\star} P<0.05,{ }^{* *} P<0.01,{ }^{* *} P<0.001\right)$.

\section{References}

1. Stover, P. J. \& Field, M. S. Trafficking of intracellular folates. Adv. Nutr 2, 325-331 (2011).

2. Kotb, M. et al. Consensus nomenclature for the mammalian methionine adenosyltransferase genes and gene products. Trends Genet. 13, 51-52 (1997).

3. Lu, S. C. \& Mato, J. M. S-adenosylmethionine in liver health, injury, and cancer. Physiol. Rev. 92, 1515-1542 (2012).
4. Chen, H. et al. Role of methionine adenosyltransferase $2 \mathrm{~A}$ and $\mathrm{S}$-adenosylmethionine in mitogen-induced growth of human colon cancer cells. Gastroenterology 133, 207-218 (2007).

5. Liu, Q. et al. The X protein of hepatitis B virus inhibits apoptosis in hepatoma cells through enhancing the methionine adenosyltransferase $2 \mathrm{~A}$ gene expression and reducing S-adenosylmethionine production. J. Biol. Chem. 286, 17168-17180 (2011).

6. Yang, H., Li, T. W., Peng, J., Mato, J. M. \& Lu, S. C. Insulin-like growth factor 1 activates methionine adenosyltransferase $2 \mathrm{~A}$ transcription by multiple pathways in human colon cancer cells. J. Biol. Chem. 436, 507-516 (2011).

7. Zhang, T. et al. Overexpression of methionine adenosyltransferase II alpha (MAT2A) in gastric cancer and induction of cell cycle arrest and apoptosis in SGC-7901 cells by shRNA-mediated silencing of MAT2A gene. Acta Histochem. 115, 48-55 (2013).

8. Halim, A. B., LeGros, L., Chamberlin, M. E., Geller, A. \& Kotb, M. Regulation of the human MAT2A gene encoding the catalytic alpha 2 subunit of methionine adenosyltransferase, MAT II: gene organization, promoter characterization, and identification of a site in the proximal promoter that is essential for its activity. J. Biol. Chem. 276, 9784-9791 (2001).

9. LeGros, L., Halim, A. B., Chamberlin, M. E., Geller, A. \& Kotb, M. Regulation of the human MAT2B gene encoding the regulatory beta subunit of methionine adenosyltransferase, MAT II. J. Biol. Chem. 276, 24918-24924 (2001).

10. Liu, Q. et al. Silencing MAT2A gene by RNA interference inhibited cell growth and induced apoptosis in human hepatoma cells. Hepatol. Res. 37, 376-388 (2007).

11. Wang, Q. et al. Inhibition of hepatocelluar carcinoma MAT2A and MAT2beta gene expressions by single and dual small interfering RNA. J. Exp. Clin. Canc. Res. 27, 72 (2008).

12. Ramani, K. et al. Changes in the expression of methionine adenosyltransferase genes and S-adenosylmethionine homeostasis during hepatic stellate cell activation. Hepatology 51, 986-995 (2010).

13. Yang, H., Huang, Z. Z., Wang, J. \& Lu, S. C. The role of c-Myb and Sp1 in the up-regulation of methionine adenosyltransferase $2 \mathrm{~A}$ gene expression in human hepatocellular carcinoma. FASEB J 15, 1507-1516 (2001).

14. Yang, H. et al. Induction of human methionine adenosyltransferase $2 \mathrm{~A}$ expression by tumor necrosis factor alpha. Role of NF-kappa B and AP-1. J. Biol. Chem. 278, 50887-50896 (2003).

15. Frau, M. et al. Role of transcriptional and posttranscriptional regulation of methionine adenosyltransferases in liver cancer progression. Hepatology 56, 165-175 (2012).

16. Choudhary, C. et al. Lysine acetylation targets protein complexes and coregulates major cellular functions. Science 325, 834-840 (2009).

17. Lin, R. et al. Acetylation stabilizes ATP-citrate lyase to promote lipid biosynthesis and tumor growth. Mol. Cell 51, 506-518 (2013).

18. Zhao, S. et al. Regulation of cellular metabolism by protein lysine acetylation. Science 327, 1000-1004 (2010).

19. Katoh, Y. et al. Methionine adenosyltransferase II serves as a transcriptional corepressor of Maf oncoprotein. Mol. Cell 41, 554-566 (2011).

20. Allard, J. E. et al. Overexpression of folate binding protein is associated with shortened progression-free survival in uterine adenocarcinomas. Gynecol. Oncol. 107, 52-57 (2007).

21. Hartmann, L. C. et al. Folate receptor overexpression is associated with poor outcome in breast cancer. Int. J. Cancer 121, 938-942 (2007).

22. O'Shannessy, D. J. et al. Folate receptor alpha expression in lung cancer: diagnostic and prognostic significance. Oncotarget 3, 414-425 (2012).

23. Wang, Y. C. \& Chiang, E. P. Low-dose methotrexate inhibits methionine S-adenosyltransferase in vitro and in vivo. Mol. Med. 18, 423-432 (2012).

24. Jiang, W. et al. Acetylation regulates gluconeogenesis by promoting PEPCK1 degradation via recruiting the UBR5 ubiquitin ligase. Mol. Cell 43, 33-44 (2011).

25. Zhang, W. et al. Fluorinated N,N-dialkylaminostilbenes repress colon cancer by targeting methionine S-adenosyltransferase 2A. ACS Chem. Biol. 8, 796-803 (2013).

\section{Acknowledgements}

We thank Lei Zhang and Guoquan Yan from Biomedical Core Facility, Fudan University, for technical support. This work was supported by MOST (2015CB910401,

2011CB910601), NSFC (Grant No. 81430057, 31271454, 81225016), Shanghai Key basic research program (12JC1401100), '100 Talents' Program of Shanghai Health (Grant No XBR2011041), Scholar of 'Dawn' Program of Shanghai Education Commission, the Youth Science and Technology Leading Talent by MOST and Shanghai Outstanding Academic Leader (Grant No. 13XD1400600) to Q.-Y.L.

\section{Author contributions}

H.-B.Y., Y.-Y.X. and X.-N.Z. performed the experiments, analysed the data and co-wrote the manuscript. Y.Z., M.Z. and F.R. contributed to the data analysis. J.-T.L. constructed pGEX-GST-MAT II $\alpha$ plasmid and contributed data analysis. S.-W.Z. and L.-Y.W. contributed to study of human hepatocarcinoma cancer samples. Y.-Y.X. and 
S.-W.Z supervised the project. Q.-Y.L. conceived the idea, designed and supervised the study, analysed the data and co-wrote the manuscript.

\section{Additional information}

Supplementary Information accompanies this paper at http://www.nature.com/ naturecommunications

Competing financial interests: The authors declare no competing financial interests.

Reprints and permission information is available online at http://npg.nature.com/ reprintsandpermissions/
How to cite this article: Yang, H.-B. et al. Acetylation of MAT II $\alpha$ represses tumour cell growth and is decreased in human hepatocellular cancer. Nat. Commun. 6:6973 doi: $10.1038 /$ ncomms7973 (2015).

(c) (i) This work is licensed under a Creative Commons Attribution 4.0 International License. The images or other third party material in this article are included in the article's Creative Commons license, unless indicated otherwise in the credit line; if the material is not included under the Creative Commons license, users will need to obtain permission from the license holder to reproduce the material. To view a copy of this license, visit http://creativecommons.org/ licenses/by/4.0/ 